\title{
Assessment of fungal contamination in a Portuguese maternity unit
}

\author{
C. Viegas ${ }^{1}, \mathrm{R}, \mathrm{Sabino}^{2}, \mathrm{C}, \mathrm{Veríssimo}^{2} \&$ L. Rosado ${ }^{2}$ \\ ${ }^{1}$ Higher School of Health Technologies of Lisbon, \\ Polytechnic Institute of Lisbon, Portugal \\ ${ }^{2}$ National Institute of Health Dr. Ricardo Jorge, \\ Mycology Laboratory, Portugal
}

\begin{abstract}
A descriptive study was developed to monitor air fungal contamination in one Portuguese maternity. Sixty air samples were collected through impaction method. Air sampling was performed in food storage facilities, kitchen, food plating, canteen, pharmacy, sterilization areas, genecology wards, intensive care unit, operating rooms, urgency and also, outside premises, since this was the place regarded as reference. Besides air samples, forty three samples were collected by swabbing the surfaces using a 10 by $10 \mathrm{~cm}$ square stencil. Simultaneously, temperature, relative humidity and particles counting (PM10) were registered. Twenty three species of fungi were identified in air, being the two most commonly isolated the genera Penicillium (41,5\%) and Cladosporium $(28,4 \%)$. Regarding yeasts, only Rhodotorula sp. (45,2\%), Trichosporon mucoides $(51,6 \%)$ and Cryptococcus neoformans $(3,2 \%)$ were found. Thirteen species of fungi were identified in surfaces, being the most frequent the Penicillium genus $(91,6 \%)$. Concerning yeasts found in surfaces, four species were identified being Rhodotorula sp. (29,1\%) the most frequent.

There was no coincidence between prevailing genera indoors and outside premises. Moreover, some places presented fungal species different from the ones isolated outside. In the inside environment, Aspergillus species were isolated in air and surfaces. There was no significant relationship $(p>0,05)$ between fungal contamination and the studied environmental variables.

Keywords: air, surfaces, fungal contamination, environmental variables, maternity.
\end{abstract}




\section{Introduction}

The hospital environment is a potential source of infections and for this reason, knowledge of the fungal contamination in clinical settings is important for understanding possible types of nosocomial infections that may emanate from them [1]. Moreover, hospital-wide surveillance studies have shown the presence of various potentially pathogenic fungal species in health care settings [2].

The presence of fungi requires ideal conditions of temperature, humidity, oxygen, carbon sources, nitrogen and minerals. Their biological activities of biodegradation and biodeterioration depend on their enzymatic activity, environmental conditions, competition phenomenon's and nature of the substrate [3]. Regarding particles, they can be vehicle for fungal dispersion [4], favoring the spread of fungi to larger areas.

Fungal health effects are dependent on the species present, the metabolic products, the concentration and exposure duration and individual susceptibility [5], being fungal exposure in hospitals of particular interest due to the possible patient's susceptibility.

Culturing air samples is usually the only parameter used to assess indoor fungal contamination [6]. However, surfaces analysis complements the air characterization and is used in order to identify contamination sources. It may also be used in order to evaluate the efficacy of surface cleaning and disinfection [7]. Although some studies have shown differences in the frequency of fungal species isolated between air and surfaces samples, a longitudinal fungal surface survey, even if performed only two or three times a year, in definite appropriate locations and areas, can detect minor contamination and can serve as a good marker for lack of cleaning or filtration. The surveillance strategy - air and surfaces - permits the control of maintenance and cleaning procedures, the education of healthcare workers regarding infection control protocols and the definition of acceptable levels of contamination in order to introduce immediate corrective measures $[8,9]$.

Therefore, it is important to contribute to the increase of knowledge regarding to air and surfaces fungal contamination in hospitals indoor spaces in order to identify most effective preventive measures to avoid such contamination. This investigation was designed to describe environmental fungal contamination in a Portuguese maternity and to allow, if necessary, the implementation of corrective measures.

\section{Materials and methods}

A descriptive study was developed to monitor fungal contamination in the biggest Portuguese maternity during a month period. Sixty air samples were collected through impaction method. Air samples were collected at 140 $\mathrm{L} /$ minute, at one meter tall, on to malt extract agar with the antibiotic chloramphenicol (MEA), in the facilities - food storage facilities, kitchen, food plating, canteen, pharmacy, sterilization areas, genecology wards, intensive care unit, operating rooms, urgency - and also, outside premises, since this is the 
place regarded as reference. The volume of air collected indoor was 5001 and outdoor 2501 .

Besides air samples, forty three samples were collected by swabbing the surfaces of the same indoor places, using a 10 by $10 \mathrm{~cm}$ square stencil disinfected with $70 \%$ alcohol solution between samples according to the International Standard ISO 18593 - 2004.

Simultaneously, two environmental parameters - temperature and relative humidity - were monitored, using the Babouc equipment, (LSI Sistems), according to the International Standard ISO 7726 - 1998. Particles counting (PM10) were also registered.

After laboratory processing and incubation of the collected samples, quantitative $\left(\mathrm{CFU} / \mathrm{m}^{3}\right.$ and $\left.\mathrm{CFU} / \mathrm{m}^{2}\right)$ and qualitative results were obtained, with identification of isolated fungal species. Whenever possible, filamentous fungi were identified to the species level, since adverse health effects vary according to fungal species $[10,11]$. Identification of filamentous fungi was carried out on material mounted in lactophenol blue and achieved through morphological characteristics listed in illustrated literature [11] and yeasts were identified through biochemical API test [12].

Tables with frequency distribution of isolated fungal species were made with the obtained data. Fungal concentration dependence in the three monitored environmental parameters - temperature, relative humidity and particles - was also analyzed.

\section{Results}

Twenty three species of fungi were identified in the collected air samples, being Penicillium and Cladosporium the two genera most commonly found, with $41,5 \%$ and $28,4 \%$ of frequency. Regarding yeasts, only Rhodotorula sp. (45,2\%), Trichosporon mucoides (51,6\%) and Cryptococcus neoformans (3,2\%) were found. Thirteen species of fungi were identified in surfaces, being Penicillium

Table 1: $\quad$ Most frequent fungi identified in the maternity air and surfaces.

\begin{tabular}{|c|c|}
\hline Air & Frequency (\%) \\
\hline Penicillium sp. & 41,5 \\
Cladosporium sp. & 28,4 \\
Chrysonilia sp. & 10,8 \\
Aspergillus sp. & 9,1 \\
Others & 10,2 \\
\hline Surfaces & Frequency (\%) \\
\hline Penicillium sp. & 91,6 \\
Aspergillus sp. & 2,7 \\
Chrysonilia sp. & 2,0 \\
Others & 3,7 \\
\hline
\end{tabular}


genus $(91,6 \%)$ the most frequent genera. Concerning yeasts found in surfaces, four species were identified being Rhodotorula sp. $(29,1 \%)$ the most frequent.

There was no coincidence between prevailing genera indoor and outside premises. Moreover, some places presented fungal species different from the ones isolated outside. In inside environment, there were isolated Aspergillus species in air - A. ochraceus, A. versicolor, A. candidus, A. fumigatus and A. niger - and also in surfaces A. glaucus, A. terreus and A. fumigatus.

Regarding comparison of concentrations found in air, for indoor and outdoor environments, several places showed higher contamination indoor.

Concerning the influence of the monitored environmental variables temperature, relative humidity and particles - no significant correlation $(p>$ $0,05)$ was revealed.

\section{Discussion}

The mere presence of fungi in hospital air is a concern motif because many spores can be released leading to an incidence of nosocomial and occupational infections [1].

Concerning Penicillium, the predominant genus in the air and surfaces analyzed, there are different potential risks associated with their inhalation of their different species due to different the toxins release [13].

It is suggested that fungal levels found indoors should be compared, quantitatively and qualitatively, with those found outdoors, because the first are dependent on the last [5]. Nevertheless, when it comes to fungal levels, it should be taken into account that indoor and outdoor environments are quite different which, by itself, justifies diversity of species between different spaces. However, the fact that there is no stipulated limit, with regard to fungal contamination, makes it essential to compare fungal levels indoors and outdoors. Thus, indoor air quality that significantly differs from the outside air could mean that there are infiltration problems and the potential risk for health effects exists. It is worth mentioning that as outdoor air is a major source of the fungi found indoors, nonetheless there was no coincidence between prevailing genera indoor and outside premises. Moreover, some places presented fungal species different from the ones isolated outside and several places showed more contamination indoor, suggesting, all the three situations, fungal contamination from within [14], maybe due to the natural flowers and food brought for the patients [15].

Faure et al. [16] used the acceptability threshold for hospital settings $>2$ $\mathrm{CFU} /$ room without $A$. fumigatus [16]. This threshold was used to interpret air results and to perform, as soon as possible, corrective measures in the contaminated areas. Considering this threshold, from the 31 indoor environments that were monitored, $61,3 \%$ showed more than $2 \mathrm{CFU} /$ room and $6,5 \%$ presented the species A. fumigatus. Another threshold used for hospital settings, proposed by Krzysztofik in 1992 , is $200 \mathrm{CFU} / \mathrm{m}^{3}$ [17]. Regarding this threshold $9,7 \%$ of indoor places exceed this value. Macher [18] also recommended concentrations for hospital environments, such as: a) for ultra clean areas like laminar airflows a value of $<4 \mathrm{CFU} / \mathrm{m}^{3}$ (viable particle count); b) for air-conditioned areas a value 
of $<18 \mathrm{CFU} / \mathrm{m}^{3}$; c) for operation theatres and patient isolations rooms a value of $<15 \mathrm{CFU} / \mathrm{m}^{3}$ for saprophytic fungi and $<0,1 \mathrm{CFU} / \mathrm{m}^{3}$ for opportunistic fungal pathogens [18]. Regarding Matcher et al. recommendations, sterilization areas presented more than $4 \mathrm{CFU} / \mathrm{m}^{3}$ and some areas belonging to the intensive care unit presented more than $18 \mathrm{CFU} / \mathrm{m}^{3}$.

Although there is no reference for surfaces we must consider that species found in surfaces - A. glaucus, A. terreus and A. fumigatus - can be aerosolized depending on several variables, such spores dimensions [19], biological characteristics [20,21], air temperature, oxygen availability, nutrients presence [22] and also, surface vibrations [23].

Filamentous fungi were more frequent than yeasts in the maternity air and surfaces, such as in Kordbachehn et al. [24] study, made also in hospital wards [24]. However, in another studied setting - gymnasium with swimming pool was found that in surfaces the CFU counts were higher for yeasts than for fungi, which may be due to the fact that yeasts are more difficult to disseminate in the air or because they are more resistant to the products used on surfaces during the cleaning procedures [25].

Results related to environmental variables are not consistent with what is expected, because several authors showed a strict correlation between indoor fungal load and temperature, humidity and particles $[4,26]$. It was found that the relationship between the fungal air contamination and temperature, relative humidity and particles counting was not statistically significant $(p>0,05)$. This may be justified by the effect of other environmental variables also influencing fungal spreading, namely workers, visitors, food, who may carry a great diversity of fungal species [27], as well the developed activities that may also affect fungal concentration [28].

\section{Conclusions}

With this study it was possible to characterize fungal distribution in this maternity, identify eventual risk sources and most effective preventive measures to avoid such contamination.

Unlike other studies, environmental variables monitored (temperature, relative humidity and particles) did not show the expected association with fungal concentration, which may possibly have resulted from other variables not investigated in this study.

We also conclude that fungal contamination was found within the maternity wards, being this hospital setting a potential source of infections for the patients.

\section{References}

[1] Sudharsanam S., Srikanth P., Sheela M. \& Steinberg R., Study of the indoor air quality in hospitals in South Chennai, India. Microbial profile. Indoor and Built Environment, 15, 5, pp. 436-441, 2008. 
[2] Sarica S., Asan A., Otkun M. \& Ture M., Monitoring indoor airborne fungi and bacteria in the different areas of Trakya University Hospital, Edirne, Turkey. Indoor Built Environment, 11, pp. 285-292, 2002.

[3] Bursykowski T., Molenberghs G. \& Abeck D., High prevalence of foot diseases in Europe: results of the Achilles Project. Mycoses, 46, 11-12, pp. 496-505, 2003.

[4] Kim K., Park J., Kim C. \& Lee K., Distribution of airborne fungi, particulate matter and carbon dioxide in Seoul Metropolitan Subway Stations. J Prev Med Public Health, 39, pp. 325-330, 2006.

[5] Goyer N., Lavoie J., Lazure L. \& Marchand G., Bioaerosols in the Workplace: Evaluation, Control and Prevention Guide. Institut de Recherche en Santé et en Sécurité du Travail du Québec, 2001.

[6] Srikanth P., Sudharsanam S. \& Steinberg R., Bio-aerosols in indoor environment: composition, health effects and analysis. Indian Journal of Medical Microbiology, 26, 4, pp. 302-312, 2008.

[7] Stetzenbach L., Buttner M. \& Cruz P., Detection and enumeration of airborne biocontaminants. Current Opinion in Biotechnology, 15, pp. 170 174, 2004.

[8] Lebeau B., Pinel C. \& Grillot R., Infections nosocomiales fongiques et parasitaires: intérêt et limite des méthodes de désinfection. PathologieBiologie, 46, 5, pp. 335-340, 1998.

[9] Brocard-Lemort C., Normes et recommendations en hygiène environnementale hospitalière. Annales de Biologie Clinique, 58, 4, pp. 431-437, 2000.

[10] Rao C., Burge H. \& Chang J., Review of quantitative standards and guidelines for fungi in indoor air. J Air Waste Manage Assoc., 46, pp. 899 908, 1996.

[11] Hoog C., Guarro J., Gené G. \& Figueiras M., (2 ${ }^{\text {th }}$ ed). Atlas of Clinical Fungi. Centraalbureau voor Schimmelcultures, 2000.

[12] Ghannoum M., Hajeh R., Scher R., Konnikov N., et al., A large-scale North American study of fungal isolates from nails: The frequency of onychomycosis, fungal distribution and antifungal susceptibility patterns. J. Am. Acad. Dermatol, 43, pp. 641-648, 2000.

[13] Kemp P., Neumeister-Kemp H., Esposito B., Lysek G. \& Murray F., Changes in airborne fungi from the outdoors to indoor air; Large HVAC systems in nonproblem buildings in two different climates. American Industrial Hygiene Association, 64, pp. 269-275, 2003.

[14] Nevalainen A., Bio-aerosols as exposure agents in indoor environment in relation to asthma and allergy. Section 3 Asthma and allergy. Proceedings of the First ENVIE Conference on Indoor Air Quality and Health for EU Policy, Helsinki, Finland, 2007.

[15] Ekhaise F., Ighosewe O. \& Ajakpovi O., Hospital indoor airborne microflora in private and government owned hospitals in Benin City, Nigeria. Word Journal of Medical Sciences, 3 (1), pp. 19-23, 2008. 
[16] Faure O., Fricker-Hidalgo H. \& Lebeau B., Eight-year surveillance of environmental fungal contamination in hospital operating rooms and haematological units. Journal of Hospital Infection, 50, 2, pp. 155-160, 2002.

[17] Augustowska M. \& Dutkiewicz J., - Variability of airborne microflora in a hospital ward within a period of one year. Annals of Agricultural and Environmental Medicine, 13, 1, pp. 99-106, 2006.

[18] Macher J., Bioaerosols: assessment and control. Cincinnatti, OH: American Conference of Governmental Industrial Hygienists, 1999.

[19] Aydogdu, H., Asan A. \& Otkun M., Monitoring of fungi and bacteria in the indoor air of primary schools in Edirne city, Turkey. Indoor and Built Environment, 14, 5, pp. 411-425, 2005.

[20] Gomes J., Contaminação do ar interior por bioaerossóis. Revista Portuguesa de Pneumologia. VIII, 6, pp. 689-694, 2002.

[21] Lugauskas A. \& Krikstaponis A., Microscopic fungi found in the libraries of Vilnius and factors affecting their development. Indoor and Built Environment, 3, 3, pp. 169-182, 2004.

[22] Becker R., Fungal disfigurement of constructions analysis of the effects of various factors. In Samson R, Flannigan B. \& Flannigan M., Air quality monographs. Vol 2: Health implications of fungi in indoor environments. Amsterdam: Elsevier, pp. 361-380. 1994.

[23] Górny R., Reponen T. \& Grinshpun S., Source strength of fungal spore aerosolization from moldy building material. Atmospheric Environment, 35, 28 pp. 4853-4862, 2001.

[24] Kordbacheh P., Zaini F. \& Kamali P., Study on the sources of nosocomial fungal infections at intensive care unit and transplant wards at a teaching hospital in Tehran. Iranian Journal of Public Health, 34, 2, pp. 1-8, 2005.

[25] Viegas C., Alves C., Carolino E., Rosado L. \& Silva Santos C., Occupational exposure to fungi in gymnasiums with swimming pools. Fifth International Conference Environmental Health Risk, 2009.

[26] Kakde U., Kakde H. \& Saoji A., Seasonal Variation of Fungal Propagules in a Fruit Market Environment, Nagpur (India). Aerobiologia, 17, pp. 177182, 2001.

[27] Scheff P., Pulius V., Curtis L. \& Conroy L., Indoor air quality in a middle school, Part II: Development of emission factors for particulate matter and bioaerosols. Applied Occupational and Environmental Hygiene, 15, pp. 835-842, 2000.

[28] Buttner M. \& Stetzenbach L., Monitoring Airborne fungal spores in an experimental indoor environment to evaluate sampling methods and the effects of human activity on air sampling. Applied and Environmental Microbiology, 59, pp. 219-226, 1993. 